Maldonado B.

Rev. Chil. Pediatr. 66 (5); 246-250, 1995

\title{
La edad del paciente: factor determinante para la prescripción de medicamentos en las farmacias de Santiago
}

\author{
Blanca Maldonado V. ${ }^{1}$
}

\section{Over the counter prescription to infants and children}

\begin{abstract}
To look oul whether polient age influences over the couriler active drug prescriptions, simulated situclused hypothelical cases of respiratory illness affecling an infont and or a schoolage child were presented to staff of 67 pharmacies at retropolitan Santiago. Chile, by a trained inteviewer. Answers were recorded immedially after interview in specially designed forms, logether with pharriacy's neighbourhood socioeconomic level, adscription to commercicl chains of pharmacies or companies as well as gender and profession of the interviewed person. The Fisher-Irwing test for dichotomous variables was used to asses stalissical significance. In most cases patient was not referred to a physician 104/:34 177.6\%), and a diagnosis was followed by prescription of drugs in all these irstances. Anlibiolics were often prescribed $67 / 134(50 \%)$ and the visit to a phisician was considered to have been unduly posiponed in $74 / 134$ [55.2\% of coses. These outcomes were enhanced by interviewed's male gender and location of pharmacies in medium or low socioeconomic level neighbourhoods. Infants were more often referred to physicians $\{5 \mathrm{a} \%$ against $3 \%, \mathrm{p}=1.58 \times 10-8\}$ and were prescribed antimicrobials less frequently than schoolage childen 196\% against $42 \%, p=7.7 \times 1091$, an outcome that was not influenced by any other included voriable. Age less than one year seems to be a prolector factor ogainst over the couriter drug preseription in these pharmacies.
\end{abstract}

(Key words: drugs, non-prescription, pharmacies, legislation.]

En nuestro pais existe hace tiempo inquietud por medir la automedicación, estimándose a partir de estudios la información mediante encuestas a los consumidores, en muestras aleatorias $^{1.2}$ o en clientes de farmacias ${ }^{3.4}$, que el consumo de fármacos no prescritos por médicos representa entre $70 \%$ y $73 \%$ de los $\operatorname{cas}^{1,2}$, variando, al considerar sólo antibióticos, entre $22,5 \%$ y $65,7 \% 2,3$. Existe poca información sobre automedicación en los niños. En una encuesta del año 1983, la frecuencia era $47 \%$ en menores de 12 años 2 . Datos más recientes mostraron $60 \%$ global y $28,9 \%$ en menores de 15 años ${ }^{3}$. Las recomendaciones hechas por farmacias para automedicación influyen entre $16,5 \%$ y $45,7 \%$ de los casos 1,2 .

En el año 1992, con el fin de aclarar las divergencias encontradas, medir la prescripción activa de medicamentos en las farmacias de

I. Hospital Robetto del Río.
Santiago e identificar factores involucrados, se realizó una investigación en que se planté un caso clínico pediátrico en un grupo representativo de farmacias, analizándose posterionnente las conductas generadas. En la mayoría de los casos no se derivó a médico (75\%), ptescribiéndose medicamentos $(41 \%)$ y así demorando la consulta médica (24\%). La edad del consultante parecía tener un papel determinante en las conductas observadas, pero el diseño de esa investigación no permitía demostrar tal relación ${ }^{5}$. Para dilucidar hasta qué punto el comportamiento registrado había sido cauteloso por tratarse de un paciente pequeño, diseñamos otro estudio con características similares, en el cual el cuadro clínico expuesto afectara simultáneamente a un escolar y a un lactante, con la finalidad de poner en evidencia diferencias conductuales atribuibles a la edad del "paciente", esperando encontrar mayor libertad en el manejo del primer caso. Además, se pretendio indagar la influencia de otras variables. 


\section{Material y Método}

Se seleccionó la muestra basándose en datos obtenidos en un trabajo previo". que provienen de las secretarías regionales ministeriales de Santiago. Tras descartar áreas suburbanas y rurales de la periferia (Buin, Colina, Melipilla y Talagante) se identificaron 560 farmacias de Santiago jnetropolitano. que se ordenaron de acuerdo con la comuna en que se ubican, seleccionando posteriormente al azar (sistema de tómbola) el equivalente a $10 \%$ de las de cada combina, con una como mínimo. Esto permitio, además, clasificarlas según el nivel socioeconómico (NSE) atribuido a la comuna, en grupos de NSE alto (Las Condes, Viracura, Providencia, Nuñoa y la Reina) y inedio-bajo (las restantes). El segundo grupo no fue subdividido por estratos socioeconómicos porque en el esiudio anterior no se encontraron diferencias significativas entre ambos 5 . La muestra quedó conformada por 67 farmacias (12\% del tolal), ubicadas en 21 comunas de Santiago, número suficiente para detectar diferencias de $25 \%$, con erro. Tes de $5 \%$ y $20 \%$, al comparar el caso de un lactante con el de un escolar para la couducta no derivación a médico. que en el estudio previo era de $54 \%$ y se estimó como el criterio más imponante ${ }^{5,6}$.

La recolección de ínformación se realizó en dos días de septiembre de 1993 (obviando variaciones estacionales) y en horario haibil de $8: 30$ a $20: 30$ horas, que coincidia con el horario de atención en policlínicos (incluyendo ta prolongación vespertina de éste). Para evitar variaciones interpersonales. se empleś un solo encuestador quien, ininediatamente después de cada entrevista. realizada en forma verbal y directa vaciaba los datos obtenidos a un for. mulario tipo que incluia fecha y hora de la encuesta, identificacion de la farmacia. comuna, variables a estudiar y conductas observadas derivación o no a médico, emisión de diagnósticos. indicación de tratamientos y postergación explícila de la consulta médica), además de un espacio desiinado a comentarios, en el cual se consignó, entre otros, si el encuestado solicitaba más antecedentes. En cada entrevista se llenaron dos formularios correspondientes a los casos del escolar y el lactante respectivamente, con los datos obtenidos para cada uno

La encuesta se efectuó según un esquema preestablecido, que se repitió en todas las farmacias. Ante la primera persona que acudió a atender, se expuso el caso de un escolar de 6 años con tos productiva $y$ fiebre; eviando influir sobre el encuestado. Sólo en los casos que no hubo respuesta se pregunfó: ¿qué puedo hacer? (evaluando derivación a médico): ¿qué puede tener? (diagnóstico); ¿qué le puedo dar, como? (tratamiento); i.es usted (u otro) el farmacéutico? Finalizado lo anterior se le preguntó ¿no será mejor llevarlo al médico? (para evaluar retardo expreso de la consulta médiça, que defitímos como cualquier respuesta diferente de sí\}. A continuación, e independiente de lo obtenido. se comentó que el hermano de 6 meses se encontraba en iguales condiciones, reiniciando la encuesta con las mismas etapas que en la fase anterior. Exclusivamente cuando se solicitaron se aportaron otros antecedentes: evolución de dos días con síntomas catartales previos, temperatura núxima de $38,3^{\circ} \mathrm{C}$ en un solo registro en cada caso, odinofagia o furinge roja en el escolar y pesos de 6.5 y 22 $\mathrm{kg}$ respectivamente. Las variables estudiadas fueron nivel socioeconómico asignado a la comuna, pertenencia a cade- nos farmacéuticas, sexo y profesíón del encuestado (farmacéutico o dependiente). Para analizar los resultados dicotómicos. se refirieron at total de entrevistas para cada variable (134), las entnevistas por paciente para cada variable $(67$ escolar; 67 lactante) y cada variable en función de las dos edades. Se utilizaron como pruebas estadísticas la comparacion de porcentajes y la prueba de Fisher de probabilidades exactas, con $5 \%$ de nivel de signifi. cación.

\section{Resultados}

Se realizaron 134 entrevistas, 67 sobre el escolar y 67 sobre el lactante. La derivación a médico (tabla 1) ocurrió en 22,4\% (30/134), siendo afectada por la edad del caso (mayor derivacion del lactante: $58 \%$ ante $3 \%, \mathrm{p}=1,85 \cdot$ $10^{-8}$ ) y el sexo del que atendía la entrevista (menor derivación en varones: $11 \%$ vs $31 \%$, $\left.p=3,45 \cdot 10^{-3}\right)$. Cabe destacar que, aunque los despachadores de ambos sexos derivaron más frecuentemente al lactante, los varones fueron más reticentes $\left(2 \%\right.$ vs $\left.58 \%, \mathrm{p}=3 \cdot 10^{-3}\right)$, explicando la diferencia anterior. En todos los casos no derivados se emitió un diagnóstico, sjendo los más frecuentes resfrío $58 \%(60 / 134)$, bronquitis $29 \%(30 / 104)$ y amigdalitis $11 \%$ (11) 104), en similares proporciones para ambas edades y adjudicando siempre al lactante el mismo diagnóstico que al escolar. Se solicitaron antecedentes de anamnesis (tiempo de evolución, magnitud de la fiebre, etc.) en menos de $10 \%$ de las ocasiones. La formulación de diagnósticos determinó siempre conducta terapéutica $100 \%$ (104/104). Los tratamientos más frecuentemente recomendados fueron mucolíticos $55 \%$ (54/ $104)$, antigripales $20 \%(21 / 104)$, antitérmicos $5 \%$ (5/104) y antibióticos $60 \%$ (62/104). Se recomendo más de un fármaco en $40 \%$ (42/104) de los casos. No hubo concordancia entre los diagnósticos planteados y el tratamiento recomendado (v.g., bronquitis con antigripales, resfríos con antibióticos). El tratamiento indicado al lactante fue en $28 / 39$ casos el mismo que para el escolar correspondiente, pero con la mitad de la dosis, en 2/39 con la misma dosis y en los 9/39 restantes, la variación consistió básicamente en el empleo de antigripal en gotas en vez de jarabe (7/9). En 4/39 se indicaron antitusígenos con codeína. En más de $90 \%$ de las entrevistas no se solicitaron datos necesarios para la prescripción. La indicación de tratamiento se vio afectada por la edad del caso (más 
Tabla 1

Derivación a médico e indicación de tratamientos en las farmacias encuestadas, según edad del caso

\begin{tabular}{|c|c|c|c|c|c|c|c|c|c|c|}
\hline & \multicolumn{4}{|c|}{ Decivación } & \multirow{3}{*}{$\mathrm{P}^{*}$} & \multicolumn{4}{|c|}{ Prescripción } \\
\hline & & \multicolumn{2}{|c|}{ Escolar } & \multicolumn{2}{|c|}{ Lactante } & & \multicolumn{2}{|c|}{ Escolar } & \multicolumn{2}{|c|}{ Lactante } \\
\hline & & n & $\%$ & $\mathbf{n}$ & $\%$ & & $\mathbf{n}$ & $\%$ & n & $\%$ \\
\hline \multicolumn{11}{|c|}{ Sexo del encuestado } \\
\hline Femenino & $\mathrm{n}: 36$ & 2 & 6 & 21 & 58 & $9,36 \times 10^{7}$ & 34 & 94 & 15 & 4 \\
\hline Masculino & п: 34 & 0 & 0 & 7 & 23 & $5,34 \times 10^{-3}$ & 31 & 100 & 24 & 7 \\
\hline \multicolumn{11}{|l|}{ NSE farmacia ** } \\
\hline Alto & n: 23 & 1 & 4 & 12 & 52 & $3.16 \times 10^{-4}$ & 22 & 96 & 11 & 48 \\
\hline Medio-bajo & n: 44 & l & 23 & 16 & 36 & $3.10 \times 10^{-5}$ & 43 & 77 & 28 & 64 \\
\hline \multicolumn{11}{|l|}{ Profesión } \\
\hline Furnacéutico & л: 33 & l & 3 & 15 & 46 & $4,13 \times 10^{-5}$ & 32 & 97 & 18 & 54 \\
\hline Dependiense & л: 34 & I & 3 & 13 & 38 & $2,67 \times 10^{4}$ & 33 & 97 & 21 & 62 \\
\hline \multicolumn{11}{|l|}{ Tipo de farmacia } \\
\hline De cadena & $n: 16$ & 0 & 0 & 6 & 38 & $8.83 \times 10^{-3}$ & 16 & 100 & 10 & 62 \\
\hline Otras & $\mathrm{n}: 51$ & 2 & 4 & 22 & 43 & $1.82 \times 10^{-6}$ & 49 & 96 & 29 & 57 \\
\hline
\end{tabular}

* Ia no derivación determinó siempre prescripción: los valores de p son identicos.

**: nivel socineconómico de la comuna donde se encuentra la farmacia.

frecuente en el escolar: $97 \%$ ante $58 \%, p=1.85$ - $10^{-8}$ ) y el sexo del encuestado (varón: $89 \%$, mujer $69 \%, p=3,45 \cdot 10^{-3}$. Aunque varones y mujeres indicaron más frecuentemente tratamientos en el escolar, los primeros indicaron más tratamientos en el lactante ( $77 \%$ vs $42 \%$, $\mathrm{p}=3 \cdot 10^{-3}$ ).

La prescripción de antibióticos (tabla 2) también fue influida por la edad del caso (mayor para el escolar: $96 \%$ vs $42 \%, p=7,7 \cdot 10^{-9}$ y el sexo del encuestado (mayor en varones: $80 \%$ vs $60 \%, p=3,95 \cdot 10^{-2}$ ). Nuevamente ambos sexos indicaron más antibióticos al escolar, pero los varones los prescribieron más a menudo que las mujeres en el lactante $(61 \%$ vs $30 \%, \mathrm{p}=$ $\left.3,48 \cdot 10^{-2}\right)$. Algo similar ocurrí́ con el NSE, ya siendo más frecuente la prescripción en ambas categorías para el el escolar, en los establecimientos de NSE medio y bajo se prescribieron más antibióticos para el lactante que en el alto ( $52 \%$ vs $\left.6 \%, p=3,89 \cdot 10^{-2}\right)$. La consulta médica fue demorada en forma expresa en $55,2 \%$ (72/134) de los encuestados, lo que corresponde a $71 \%(72 / 104)$ de las farmacias en que se indicaron tratamientos. La demora fue mayor en el lactante $\left(32 / 39\right.$ vs $42 / 65, p=4,48 \cdot 10^{-2}$ y fue afectada por la profesión del encuestado. La mayor demora ocurrió para el escolar, el encuestado varón y la ubicación en NSE mediobajo de la farmacia (tabla 3).

\section{Comentario}

Estos resultados son similares a los encontrado en otros lugares ${ }^{7.8}$, muestran que el problema es más complejo de lo que aparenta en primera instancia. La tendencia a automedicarse se potencia por lo que parece mayor liberalidad en la indicación de fármacos por personal no médico.

En una encuesta sobre el consumo de medicamentos en Santiago ${ }^{2}$, las proporciones de prescripciones por recetas médicas eran similares a las obtenidas por otras fuentes ( $48 \%$ vs $47 \%$ ) y, si bien la experiencia del paciente es un factor preeminente en la automedicación $(76,9 \%)$, la influencia de recomendaciones hechas por otros (farmacias, familiares y amigos, enfermeras $y$ practicantes) no es despreciable $2,3,9$, pese a la 


\section{Tabla 2}

Prescripción de antibióticos en las farmacius encuestadas según edad del caso

$\begin{array}{ccc}\text { Prescripción de antibiúticos } \\ \text { Escolar } & \text { Lactante } \\ n^{*} \quad \% & \text { p* } \%\end{array}$

Scxo del encuestado

$\begin{array}{llrrrr}\text { Femenino } & (26 / 28) & 93 & (9 / 30) & 30 & 6,33 \times 10^{-} \\ \text {Masculino } & (17 / 17) & 100 & (11 / 18) & 61 & 4,73 \times 10^{-7}\end{array}$

NSE farmacia ${ }^{* *}$

$\begin{array}{llllll}\text { Alto } & (12 / 13) & 92 & (3 / 15) & 20 & 1,60 \times 10^{-4} \\ \text { Medio-bajo } & (31 / 32) & 97 & (17 / 33) & 52 & 2,06 \times 10^{-5}\end{array}$

\section{Profesión}

Farnacético $(22 / 23) \quad 96 \quad 19 / 24) \quad 3 \overline{5} \quad 2.04 \times 10^{-5}$ Dependiente $(21 / 22) \quad 96(11 / 24) \quad 48 \quad 2,37 \times 10^{-1}$

Tipo de furmacia

$\begin{array}{lrrrrr}\text { De cadena } & (6 / 6) & 100 & (4 / 10) & 6 & 2.62 \times 10^{2} \\ \text { Otras } & (37 / 39) & 95 & (16 / 38) & 48 & 3.12 \times 10^{7}\end{array}$

: : en cada ucasión se comparan los casos que recibieron antibinticos ton los no tratidos.

** : nivel socioeconómico de la conuma donde se encuenIra la farmatia.

legislación sanitaria vigente ${ }^{10}$. Afortunadamente esta liberalidad no es tan acentuada para con el lactance menor, prefiriéndose generalmente derivarlo a médico o. al menos, demostrando cierta cautela al prescribirle medicamentos.

Como en otros estudios ${ }^{2}$ se encontró una relación inversa entre nivel socioeconómico de la comunidad y autonedicacion, sobre cuyas causas sólo podríamos especular, entre otras. supuesto menor riesgo de acciones legales. en NSE mós bajos, ante acciones terapéuticas por personal no médico.

En un trabajo anterior ${ }^{5}$, los despachadores varones derivaban a médico con menor frecuencia, to que fue atribuido a una mayor tendencia a adoptar el rol de médico. En este estudio, si bien sc mantiene el fenómeno, las encuestadas mujeres sólo dificren para el lactante, en quien también prescriben con mayor frecuencia antibióticos, mientras en los escolares sólo condicionaria mayor retardo de la consulta a médico. Tal vez la diferencia en esta conducta proviene de las mujeres, que por asumir más frecuente- mente la responsabilidad directa de la salud de los hijos, pudiesen estar más sensibilizadas a los riesgos de hacerlo en forma indebida, adoptando una conducta más precavida, especialmente en el lactante. Como no se encontraron diferencias en el retardo de la consulta médica para las dos edades, es posible que, si bien la edad menor de un año influye en la decisión de dar o no tratamiento, una vez tomada la de hacerlo ésta no se modifica. Es difícil explicar la demora de la consulta médica causada por farmacéuticos. lo que talvez se deba a confusiones del rol que deben desempen̆ar las farmacias en el sistema de salud ${ }^{3,4,8}$ y cuyas razones requieren más estudio.

En conclusión, en la mayoría de las farmacias investigadas no se derivó a médico, formulándose diagnósticos que conllevaron tratamientos, frecuentemente antibióticos, siendo común el retardo en la consulta médica. Estas conductas se acentúan si el despachador es varón, si la

\section{Tabla 3}

Demora en la consulta médica en las farmacias que indican tratamiento

Demora en la consulta médica
Escolar Lactente
$\mathrm{n}^{*} \%$

Sexo del elicuestado

$\begin{array}{llllll}\text { Femenino } & (17 / 34) & 50^{* *} & (11 / 15) & 73 & 0.21 \\ \text { Masculino } & (25 / 35) & 81^{* \prime} & (21 / 24) & 88 & 0,38\end{array}$

NSE farmacia ${ }^{* * *}$

$\begin{array}{lllrrr}\text { Alto } & (10 / 22) & 46^{* *} & (8 / 11) & 73 & 0,13 \\ \text { Medio-hajo } & (32 / 43) & 74^{*+} & (24 / 28) & 86 & 0,20\end{array}$

\section{Profesión}

$\begin{array}{llllll}\text { Farmacéutico } & (20 / 32) & 45 & (16 / 18) & 89 & 4.40 \times 10^{-2}\end{array}$ Dependiente $\quad(22 / 33) \quad 67 \quad(16 / 21) \quad 76 \quad 0,3.3$

Tipo de farmacia

$\begin{array}{llllll}\text { De cadella } & (10 / 16) & 63 & (8 / 10) & 80 & 0,31 \\ \text { Otras } & (32 / 49) & 65 & (24 / 29) & 83 & 7,95 \times 10^{-2}\end{array}$

- : coroo la indicación de tratamientos voría al considerar la edad, los n son variables

* : para el escolas deternina nlayor demora el sexo masculin, del encuestado $\left(p=9.5 \times 10^{-3}\right)$ y el NSE nıdıo-bajo $\left(\mathrm{p}=2.14 \times 10^{\circ}{ }^{2}\right\}$

***: nivel socioeconómico de la comuna donde se encuentra la farınacia. 
farmacia se ubica en comunas de nivel socioeconómico medio-bajo. La única variable, entre las investigadas, que parece limitar dichas conductas es la edad menor de un año.

\section{Resumen}

Para averiguar si la edad de los niños influye en la prescripción de medicamentos por parte de las farmacias, se encuestaron los dependientes de 67 de estos establecimientos en distintas comunas de Santiago, Chile, mediante una consulta simulada sobre una enfermedad respiratoria en un niño de edad escolar y un lactante. Las respuestas se registraron en formularios tipo, con el nivel socioeconómico (NSE) asignado a la comuna del establecimiento, pertenencia a cadenas farmacéuticas, sexo y profesión del encuestado. La evaluación estadística de resultados dicotómicos incluyó prueba de FisherIrwing. No se derivó a médico $77,6 \%$ (104/134) de las consultas, formulándose diagnósticos en todos estos casos (104/104), los que siempre originaron prescripción de medicamentos, frecuentemente antibióticos $50 \%(67 / 134)$, dilatándose la consulta médica en $55,2 \%$ (74/134) de los casos, lo que fue más acentuado si el dependiente era varón y la farmacia estaba en un barrio de NSE medio-bajo. El lactante fue más frecuentemente derivado a médico ( $58 \%$ vs $3 \%$, $\left.\mathrm{p}=1,85 \cdot 10^{-8}\right)$, prescribiéndosele menos veces antibióticos ( $96 \%$ vs $\left.42 \%, p=7,7 \times 10^{-9}\right)$, independientemente de todas las demás variables investigadas. La edad menor de un año constituye un factor limitante a la prescripción directa de medicamentos en las farmacias encuestadas.

(Palabras clave: medicamentos, no prescritos, farmacias, legislación.)

\section{Referencias}

1. Baldini $R$, yarbera $A$ : Uso de medicamentos sin prescripción médica un estudio epidemiologico. Rev Med Valparaíso 1979: 32: 55-58.

2. Sánchez E. Nazal M. Saldtas C, Raempfer AM: Encuesta sobre el consumo de medicamentos en el Gran Santiago. Rev Med Chil 1984; 112; 185-191.

3. Damhier A. Brieva J. Yales $T$. Villegas G. Pérez $H$ : Prescripción y dispensación de sulfonamidas antisépticos urizarios y antiparasitarios en Concepción, Chile. Bol Oficina Sayit Panam 1984; $97: 478-488$.

4. Frias $x$, Cordano $G$, Chávez $H$ : Estudio sobre las consultas del público en farmacias de Santiago. Actual Farmac 1992: 49: 10-13.

5. Maldonddo B, Cabrera $C$, Soto $D$ : Prescripción de medicameatos en las farmacias del Gran Santiago: un problems subesttmado (Resumen). Rev Chil Pediatr 1992; 63 (volumen especia) : 75+76.

6. Fleias JL: Staristical methods for rates and proportions. 2' ed. New York, John Wiley and Sons 1972: $260-280$.

7. Snibelmant M. Ramos L. Fermandes A. Pecis $D$. Sarmento 5: Indiçāo de medicamentos por balcotistas de farmacias de Porto Alegre. AMB Rev Assoc Med Brasil 1986; 32: 79-83.

8. Compos I, Sabino J. Martins D. Dulton C: Prescrito de medicamentos por balconistas de 72 farmacias de Belo Horizonte. J Pediatr (Rio de Janciro) 1985; 59: 307-312.

9. Brieva J, Danhier $C$, Yates $T$, Villegas $G$, Pérez $C$. Características de la prescripción y dispensación de antibióticos en la ciudad de Concepción, Chile. Bol Oficina Sanit Panam 1983; 261-269.

10. Chile. Ministerio de Salud. Reglamento de farmacias, droguerias, almacenes farmacéticos y botiquines autorizados. Decreto Supremo Nímero 466 del 31 de diciembre de 1984. Samliago, Chile. 